\title{
Joined Greenhouse Environment Control System Based On ZigBee
}

\author{
Yanmei Tan, Chaoning Jiang
}

Guangxi Technological College of Machinery and Electricity (GXCME), Nanning, 530007, China

Keywords: Joined Greenhouse; Environment Control; ZigBee; GPRS/ WiFi

\begin{abstract}
According to the environment control of the joined greenhouse, one method that about the monitoring and intervention of the greenhouse environment with intelligent mobile phone and computer has been proposed. Firstly, the basic structure of the system has been analysed, several short and medium range wireless technology are compared; then, the field data collection based on ZigBee and the monitoring scheme about the network coordination and management communication based on GPRS/WiFi has been determined, and described the system from the part of the software and hardware that reflects the confirming of the system. At last, prospect of the greenhouse environment control perspective with the latest technology, and do some research on the next stage of work.
\end{abstract}

\section{Introduction}

Man-made greenhouse is helpful to improve the agricultural production facilities and one of the modern agricultural development direction. At present, the internet of things in the monitoring of greenhouse has showed great application value, and widely used in agricultural production factory [1], in greenhouse was basically realized low cost, high benefit, ecological and environmental protection, and easy management goals. From the point of the architectural form, mainly divided into pavilions in greenhouse and in greenhouse. In greenhouse was compared with the former, not only can save land, building materials, has a considerable price, and there are large internal space, easy to realize centralized management. According to statistics, from 2004 to 2014, the area of the greenhouse in China rose nearly 2 times, therefore, the pursuit of low cost, high benefit, ecological and environmental protection, easy to manage and regulate the system of greenhouse technology and facilities are more widely used is the current of the premision.

\section{The greenhouse environment control system overview}

Greenhouse environmental control system mainly realize the indoor temperature, humidity and other parameters for automatic monitoring, processing, real-time control, such as online intervention [3]. For ease of main control computer and several measuring sensor in greenhouse, execute, two-way and multipoint data communication between modules, information flow medium for the spread of the data and information management and control is the guarantee of the premise, based on the differences of stream media, mainly divides into the cable transmission and wireless transmission.

In the environment of greenhouse control system is based on ZigBee, different in greenhouse (1, $2, \ldots \mathrm{n})$ greenhouse environmental information (humidity, temperature, etc.) by the sensor, the ZigBee transmission to the Sink node, and then by the Sink node will be measured by the greenhouse environment parameter uploaded to the PC, according to the transmission of greenhouse environment parameters, the corresponding greenhouse to intervene, then adjust the greenhouse environment.

Between the greenhouse control system about ZigBee is applicated in greenhouse environment to distinguish the mainly concentrated in the Sink node of wireless sensor network and the communication between upper machine[5], this paper proposes a system structure of "dispersionconcentrated", decentralized ZigBee sensor nodes by the embedded module together as sensor networks, the ZigBee sensor node parameters of greenhouse environment can be measured by the network transmission to the embedded module, and then by the embedded module through GPRS 
communication transfer real-time data information to the remote computer, intervention by monitoring center, the greenhouse environment to adjust; Using ZigBee - [6] WiFi gateway instead of embedded module for sensor network connection, to realize the collection of greenhouse environment parameters and remote monitoring; [7] put forward $3 \mathrm{~g}$ network as data information transmission network, the user can through the mobile phone or computer to monitor greenhouse environment parameter; Bluetooth is given [8] as the main, from the machine with the connected sensor network and PC.

\section{The system structure and the parts}

\section{System basic structure}

Sink node system uses to CC2430 ZigBee module as the core, to highlight the central role of ZigBee can be "a greenhouse" for example. Temperature "greenhouse 1", humidity, light, soil information sent via ZigBee, regulation of greenhouse environment to perform terminal hair through ZigBee.

\section{All the parts of thesystem}

(1) The terminal module. In greenhouse of the terminal including data acquisition, control and execution module. Data collection includes information such as temperature, humidity, illumination, soil; Control and execution unit is according to the greenhouse and plant growth conditions for intervention, for example, the weak light intensity, temperature in greenhouse is lower than the limit plant growth requirements, at this point, according to the collected data, monitoring personnel or master computer control instruction, via wireless transmission, to the "1" greenhouse control and execution module, the shutter motor control and temperature control equipment. Various sensors were constituted by the scene in greenhouse subnet, execute equipment such as roller machine belongs to the controlled object, through to the inside the greenhouse temperature, humidity, illumination, soil, such as information acquisition, linkage control related equipment can be directly or send the information to the management, decision-making, intervention in greenhouse environment adjustment.

(2)The Sink module. In the greenhouse environmental control system, Sink module on the one hand, is responsible for receiving information acquisition in greenhouse parameters and to transfer to the management to provide effective way, on the other hand for each site subnet coupling connection, form a wireless sensor network. For this purpose, the Sink module is also known as network coordination module, its core is based on CC2430 data receiving and sending unigt.

(3) The remote monitoring. The remote monitoring, according to the difference of management object is divided into smartphone management based on GPRS and PC monitoring based on WiFi. Due to the management of liquidity, the use of smart phones through GPRS access management page, able to understand anywhere inside the greenhouse environment. Dueto considering the greenhouse live far away from the control room (over 100 meters), WiFi PC monitoring, more conducive to the accumulation of plant growth model, the accumulation of the greenhouse management experience [9].

\section{The analysis of system of hardware and software part}

\section{The hardware part}

For example, the hardware part of the system includes acquisition and implementation, coordination and management of three parts in the first "greenhouse", as shown in figure 1.

(1) Greenhouse parameters acquisition and intervention. Greenhouse parameters acquisition and intervention is built on the basis of field terminal CC2430. Greenhouse temperature acquisition, for example, can use small volume, low cost, easy to use and encapsulated DS18B20 digital temperature sensor. Different nodes acquisition corresponding DS18B20 temperature value, through the ZigBee real-time transmission to the network coordinator, and then upload the data for management to monitor the greenhouse temperature, timely intervention. 


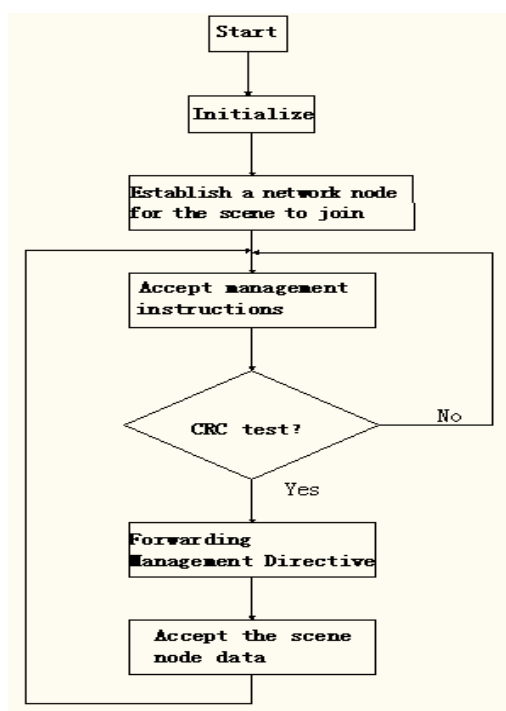

Fig.1 Hardware Structure

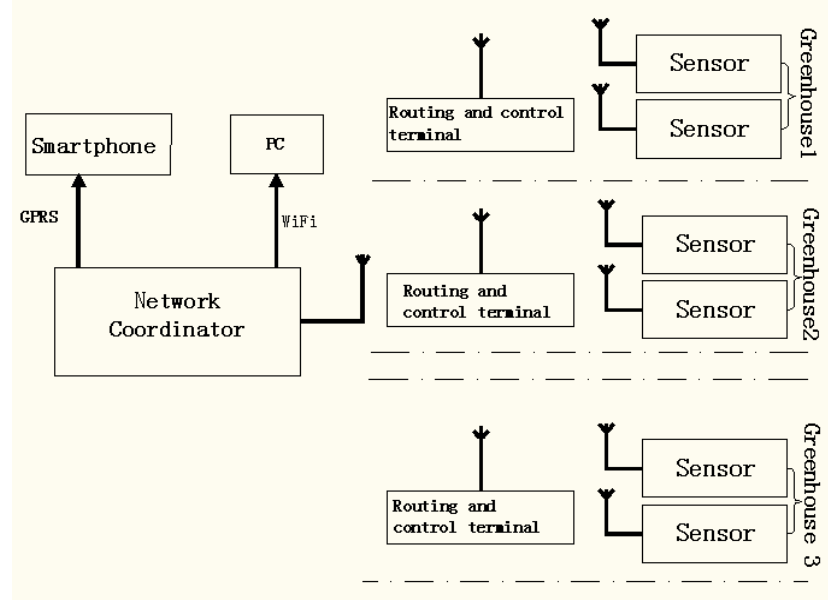

Fig.2 Network Coordination Software Process

(2) The network coordination management. Network coordination management is responsible for the network and the testing data from the terminal, and through Wi-Fi router to industrial PC.

(3) Access to data management and monitoring. Access to data management and monitoring is one of the core of this system. When the Android phone in wi-fi router radio range, Sink node (coordinator) and wi-fi router transmission of information flow to the upper machine android phone. Considering the actual coverage, Android phones in the remote, is beyond the scope of wi-fi router broadcasts, need use GPRS data access to the Web server. Use GPRS on greenhouse parameters real-time access, through the ZigBee environment parameter of data collection, stored in a data center server, and then use GPRS operators, real time control of the environmental conditions in greenhouse can be on the phone.

\section{Software}

Greenhouse control system software part includes the greenhouse environment parameter control node program, network program coordinator and the upper machine three parts, the network control program coordinator and node is the core of the realization of greenhouse control, the process as shown in figure 2, 3, respectively. In figure 6, first of all, the network coordinator initialization, data network, to create an acceptable when there is data need to transport, the data is forwarded. 


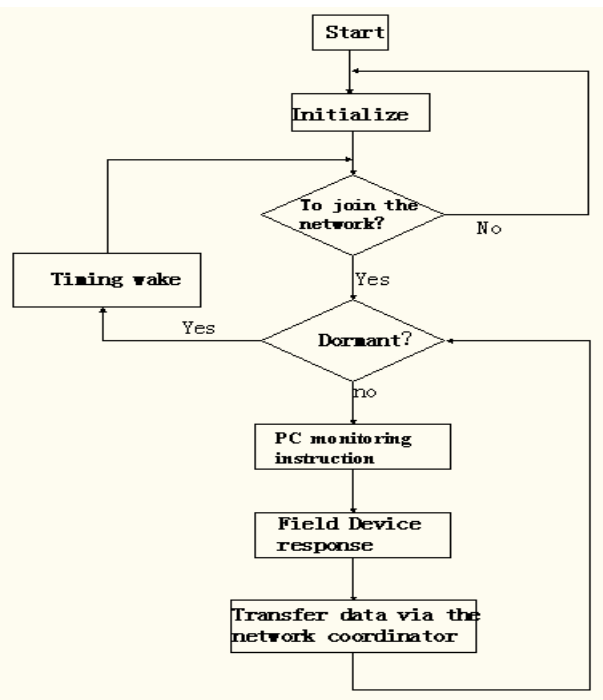

Fig.3 Field Data Collection Software Process

In figure 3, all greenhouse parameters acquisition node of the network is consistent with the [10]. Field terminal receives the coordinator via the network transmission of PC monitoring instruction, terminal sensors to collect data, and actuators to respond, then at the scene of the greenhouse is uploaded to the management.

The greenhouse environmental control system is inadequate and prospect relative to the cable, wireless control of greenhouse environment in line with the requirements of agricultural development. But in the wireless control, because there are some differences in greenhouse environment, detection sensor is a kind of high precision, wireless node layout is an important topic in greenhouse, in addition, to expand the application of greenhouse wireless control means, consider regional distribution of agricultural greenhouse, the primary focus is to monitor the cost and the network.

\section{The feasibility of building}

In addition, with the development of new technology, especially in the agricultural expert system, such as the development, a corresponding crop growth model of the expert system for collaborative management regulation is one of the important developing direction of greenhouse management and regulation.

\section{Summary}

At the scene of the greenhouse parameters acquisition and inside the greenhouse environment control is a complicated system engineering. For now, the greenhouse environment management and control is still in its stage of development, we need to make unremitting efforts. At the same time, this paper analysed a for remote (over 100 meters), the use of ZigBee parameter acquisition of greenhouse environment control system on the spot, it: (1) according to different management state of observation, using both WiFi and GPRS technology, respectively to adjust and control of greenhouse environment; (2) the parameters through the wireless network nodes by the network coordinator to management, management of the intervention of the instructions are sent to the corresponding field devices can be coordinated via the network node, adjusted to the environment of greenhouse.

\section{References}

[1] Yao Shifeng Feng Chungui, He Yuanyuan, etc. The application of Internet of things in agriculture [J]. Journal of agricultural mechanization research, 2011, (7): 190-193. 
[2] Shandong facility vegetable cultivation technology reached the international advanced level [J]. Journal of agricultural knowledge, 2011, (5): 46 and 47.

[3] duchamp, ying-xia li, Ma Chengwei, etc. The greenhouse environment control system hardware research progress [J]. Journal of agricultural engineering, 2004, 20 (1): 7-12.

[4] xiao-jing li, Zhang Kan oracle. WenShiQun based on CAN bus control system design and implementation of [J]. Computer engineering, 2010, 36 (1): 245-246.

[5] jian-wei, ZuoZhong Zou Qiuying. Agricultural remote monitoring system based on ZigBee and embedded technology design [J]. Journal of Chinese agricultural mechanization, 2010, 6 (232): 8386.

[6] wei-bo zhong wang tingting, ze-wu zhang. The environment of intelligent sensor system based on ZigBee and WiFi development [J]. Journal of agricultural mechanization research, 2012, (12): 186-189.

[7] ChengPing Guo Yangyang, ping-ping li. Based on ZigBee and $3 \mathrm{~g}$ technology of facility agriculture intelligent measurement and control system [J]. Journal of agricultural machinery, 2012, 43 (12): 229-233.

[8] CAI Ken, ke-qiang wang, YueHongWei, etc. The farmland information monitoring and bluetooth wireless transmission system design [J]. Journal of agricultural mechanization research, 2012, (1): 80-83.

[9] Ulam S. On Some Mathematical Properties Connected With Patterns Of Growth Of Figures[C]. American Mathematical Society, PSAM, 1962: 215-224.

[10] Pan Dayu. ZigBee technology in agricultural research and application of automatic monitoring and control system [D]. Qufu: master degree theses of master of qufu normal university, 2009. 